\title{
Tapered photonic crystal fibers coated with ultra-thin films for highly sensitive bio-chemical sensing
}

\author{
Vladimir P. Minkovich ${ }^{1 *}$ and Alexander B. Sotsky ${ }^{2}$
}

\begin{abstract}
Background: Photonic crystal fiber Mach-Zehnder modal interferometers based on no adiabatic tapered fibers are perspective for bio-chemical sensing, since they have very high refractive index sensitivity and it is possible for them to use a very small quantity of investigated samples. To obtain a desirable sensitivity to a needed analyte, it was proposed to coat a sensing surface of the refractive index sensor with ultra-thin films.

Methods: In this work, we reported on a no adiabatic tapered special photonic crystal fiber coated with an ultrathin layer of a Bovine Serum Albumin (BSA) antigen or with an $8 \mathrm{~nm}$ palladium film to detect interaction between the BSA antigen and an anti-BSA antibody or for fast detection of hydrogen concentrations, respectively.

Results and conclusion: During our experiments, we received a record detection limit of $125 \mathrm{pg} / \mathrm{ml}$ of the antiBSA antibody concentration. Fast detection of hydrogen concentrations up to 5.6 vol\% was carried out. By using a proposed electrodynamics model of a hydrogen sensor, an optimal taper sensing length was determined.
\end{abstract}

Keywords: Optical fiber devices, Optical fiber sensors, Optical fiber interferometer, Photonic crystal fibers

\section{Background}

Photonic crystal fiber [1] Mach-Zehnder modal interferometers (PCF MZMIs) based on no adiabatically tapered silica PCFs [2, 3] first were used for a high-resolution refractive index sensing of liquids with indices ranging from 1.33 to 1.45 [2]. It is also known that the PCF MZMIs are temperature-independent up to $180^{\circ} \mathrm{C}$ [4]. PCF MZMIs based on no adiabatically tapered fibers are attractive for bio-chemical sensing, because they can have a very small sensing length $[2,3]$. To obtain a specific sensitivity for a chosen analyte in a complex composition, it was proposed to coat a sensing surface of a refractive index (RI) sensor with a layer of an active component, characterized with a high affinity to the chosen analyte.

\section{Sensor fabrication and operating mechanism}

We employed for fabrication of our tapers a home-made PCF consisting of a solid core surrounded by four rings of

\footnotetext{
* Correspondence: vladimir@cio.mx

${ }^{1}$ Centro de Investigaciones en Optica, Division of Photonics, Loma del Bosque 115, Colonia Lomas del Campestre, 37150, Leon, Guanajuato, Mexico Full list of author information is available at the end of the article
}

air-holes in the cladding arranged in a hexagonal pattern [5]. A micrograph of our PCF cross section is presented in Fig. 1 (left). We used a PCF with the following parameters: outer diameter of $125 \mu \mathrm{m}$, core diameter of $11 \mu \mathrm{m}$, average hole diameter of $2.7 \mu \mathrm{m}$, average hole spacing (pitch) of $5.45 \mu \mathrm{m}$. To fabricate a taper, the fiber is heated and stretched. The waist of the PCF (the thinnest part of a taper) are reduced until the air hole collapse [2], obtaining a piece of a solid fiber with the diameter $\rho_{W} \sim$ less than $33 \mu \mathrm{m}$. The solid fiber can support multiple modes (waist modes). The beating of these modes inside the solid fiber is sensitive to an external environment. The resulting fundamental mode in the final transition zone (right part of Fig. 1) carries the interference information generated at the tapered zone to the detector. We consider for simplicity only two waist modes for the interference with effective refractive indices, $n_{1}$ and $n_{2}$. In this case, the taper intensity output is [6]:

$$
I_{t}=I_{1}+I_{2}+2 \sqrt{I_{1} I_{2}} \cos (\Delta \theta)
$$

where $I_{1}$ and $I_{2}$ are the intensities of the interactive 

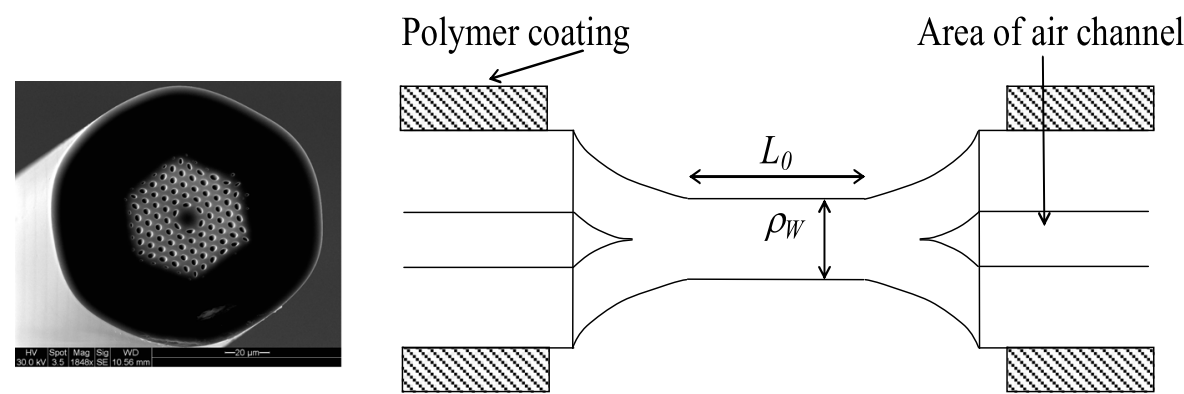

Fig. $1 \mathrm{~A}$ micrograph of the PCF cross section (left) and an illustration of the tapered PCF (right). $L_{0}$ is the length of the waist and $\rho_{W}$ is the waist diameter [6]

modes, respectively, and $\Delta \theta$ is the phase difference between them.

This phase difference depends on the difference between the effective mode refractive indices $\left(\Delta n=n_{1}-n_{2}\right)$ and the length of the waist $L_{0}$ :

$$
\Delta \theta=\frac{2 \pi}{\lambda}(\Delta n) L_{0}
$$

where $\lambda$ is the central wavelength of the light source used. The maxima of the interferometer transmittance will appear at $2 \pi \Delta n L_{0} / \lambda=2 \pi m$, where $m$ is an integer. A change of refractive index of a surrounding medium can be visualized as a shift in the output spectrum pattern [3]. We found that additional losses at tapering of our PCF were typically less than $3 \mathrm{~dB}$.

We implemented for testing of our tapers a setup consisting of an LED with peak emission at $1550 \mathrm{~nm}$ (or $1280 \mathrm{~nm}$ ) and about $80 \mathrm{~nm}$ of spectral width and an optical spectrum analyzer (OSA), Ando AQ-6315E. Usually, about $30 \mathrm{~cm}$ of the PCF were fusion spliced between standard single-mode fibers (SMF-28). We fabricated a PCF tapered section by heating of a PCF section with an oscillating high-temperature flame torch and slowly stretching it. For PCF tapering, a Vytran GPX3400 also can be used. In all experiments the tapered PCF section was held straight.

\section{Results and discussion} Highly sensitive bio-sensing

For our biosensor experiments, we used a PCF taper with a waist length $L_{O}=10 \mathrm{~mm}$ and a waist diameter $\rho_{W}=18.1 \mu \mathrm{m}$. To fabricate the taper, we applied a Vytran GPX3400 glass processing machine. The pulling speed at tapering the fiber was kept at $1 \mathrm{~mm} / \mathrm{s}$ and the heat was arranged at $90 \mathrm{~W}$. An LED source with a center wavelength of $1550 \mathrm{~nm}$ and an OSA, Ando AQ-6315E, were used to make the SM-PC-SM fiber transmission spectrum measurements. A special work cell was designed with a working volume of $50 \mu \mathrm{l}$ and a cavity length of $13 \mathrm{~mm}$ to measure small volumes of investigated solutions with our fiber sensor. Before biosensor experiments, we tested a sensitivity of our device for bio-sensing RI range using a sodium chloride $(\mathrm{NaCl})$ diluted in a distilled water in the following concentrations, $0.0,0.2,0.4,0.6,0.8$, and $1 \mathrm{M}$. Calculated refractive indices of investigated solutions at 1550 are, respectively, 1.30864, 1.31104, 1.31339, 1.31569, 1.31794, and 1.32014 [8]. Figure 2(a) shows that transmission peaks shift to longer wavelength as the refractive indices of the $\mathrm{NaCl}$ solutions increase. For the analysis of the peak shifts we selected a peak 1 . Correlation of the peak 1 maxima (between 1538 and $1548 \mathrm{~nm}$ ) with the respective RI of investigated solutions is shown in Fig. 2(b).
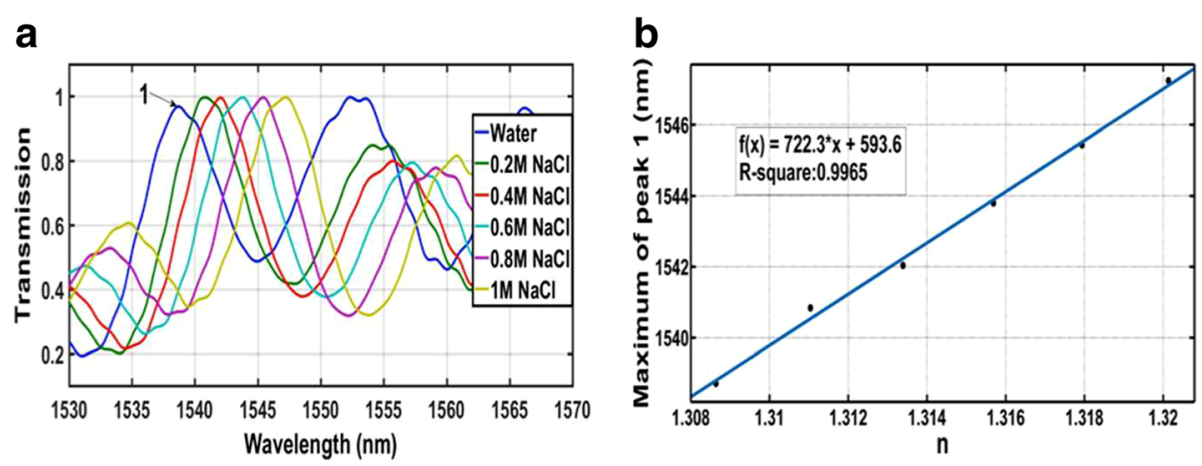

Fig. 2 (a)Transmission spectra of the sensor at different concentrations of $\mathrm{NaCl}$, (b) linear fit of the sensor response for different concentrations of $\mathrm{NaCl}$ with RI between 1.30864 and 1.32014 (reprinted with permission from Ref. [7], IEEE/OSA 
It is possible to see that we achieved a sensitivity of $722.3 \mathrm{~nm} / \mathrm{RIU}$ (with a linearity of 0.9965 ). Such a sensitivity can be compared with other sensors known. To evaluate the performance of our device for a biosensor application, antigen-antibody tests were carried out. The bovine serum albumin (BSA) antigen was chosen in our experiments. The immobilization process (covalent binding between the BSA antigen and the activated tapered silica surface was conducted like the one presented in [9]. The activation of the taper surface with an aminosilane APTES was carried out preliminary. The biosensor tests were conducted at room temperature (about $25^{\circ} \mathrm{C}$ ) by using the (anti-BSA) diluted in phosphate-buffered saline (PBS) buffer at different concentrations $(125,12.5$, 1.25 , and $0.125 \mathrm{ng} / \mathrm{ml})$. We conducted the following procedures. Step 1: Application of the tris (hydroxymethyl) (TRIS) buffer for 3 min to clean the taper surface. Step 2: Removal of the TRIS buffer, application of the PBS, and making a measurement of the optical spectrum that corresponds to the baseline. Step 3: Removal of the PBS buffer, application of the desired anti-BSA solution, and taking measurements for $10 \mathrm{~min}$. We have found in our previous experiments that after about $8 \mathrm{~min}$ of interaction the variation of shifts can be neglected. The procedures were repeated for samples with different concentrations of anti-BSA. The difference in a wavelength shift between a baseline peak and the same peak, obtained after $8 \mathrm{~min}$ of sample interaction, we related to the sample concentration. The results of our biosensor application, where the magnitude of the response is approximately proportional to the anti-BSA concentration is shown in Fig. 3.

It is possible to see in Fig. 3 that by using our sensor it is possible to measure very low anti-BSA concentrations, with a detection limit of $125 \mathrm{pg} / \mathrm{ml}$, which is lower, for example, than the similar one reported in [10]. Kinetics of BSA antigen-antibody interaction is seen. It is necessary also to point out that our sensor is capable to detect a specific protein inside a complex sample (with different protein solutions). The increased sensitivity in our case is received, since the BSA molecules were immobilized onto the taper surface that has exponentially higher IR sensitivity than areas farther away. The proposed sensor also has a much lower detection limit comparing with those reported in [11, 12], which have detection limits of $10 \mathrm{ng} / \mathrm{ml}$ and $1.1 \mathrm{ng} / \mathrm{ml}$, respectively. It was found that the estimated maximum resolution of our sensor was around $1 \times 10^{-2} \mathrm{ng} / \mathrm{ml}$, considering resolution of our spectrum analyzer of $0.05 \mathrm{~nm}$. It is necessary to point out that at using an OSA with a higher resolution an enhanced resolution of our measurements is also possible. For concentrations of anti-BSA between 0.125 and $125 \mathrm{ng} / \mathrm{ml}$ we did not have any saturation in a sensor response, see Fig. 3. It is also possible to see in this figure that the spectral shift has highly nonlinear dependence on the anti-BSA concentration. It is necessary to remind that in Fig. 2 we received a linear dependence of our sensor response, when any chemical binding between $\mathrm{NaCl}$ solutions and a silica surface was absent. That means, the BSA-antibody binding process has a great impact on our sensor response.

\section{Hydrogen detection}

The evanescent fields of PCF propagating waist modes can be changed with different ultra-thin films deposited on a PCF taper. If refractive or absorption indexes of the films are changed under action of external environment, output spectra of a PCF taper will be also changed. In this section the sensing of hydrogen is demonstrated. It is known that hydrogen is explosive in air at a room temperature and pressure at concentrations starting from about 4 vol.\% [13]. So, for safety reason it is

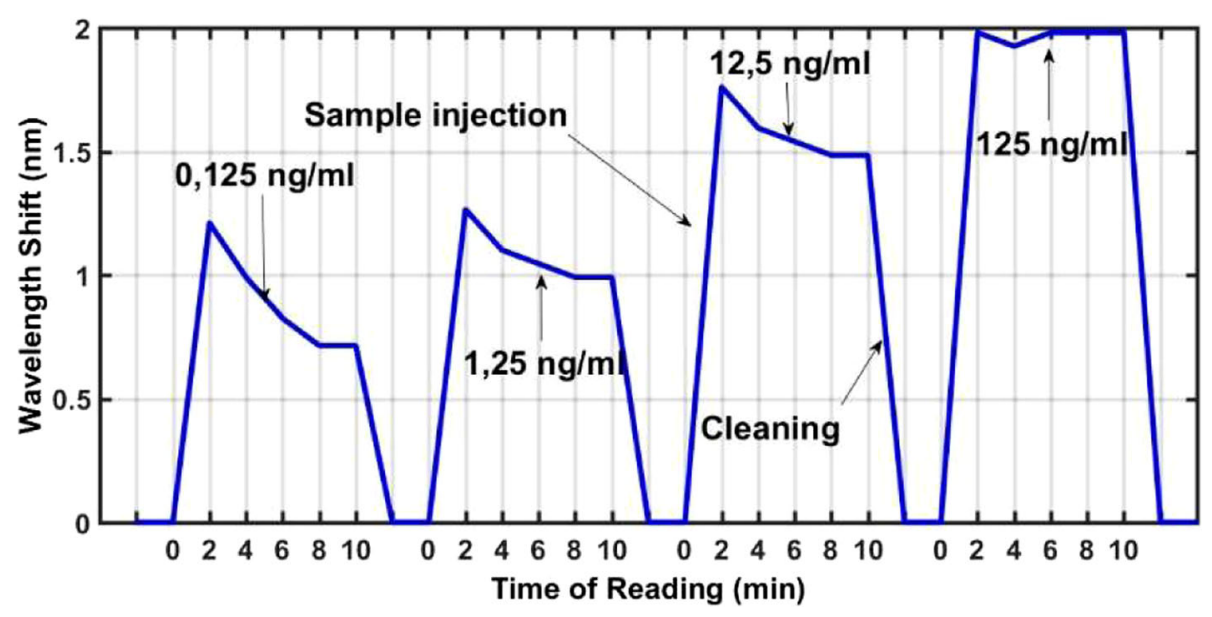

Fig. 3 Sensor response for concentrations of anti-BSA between 0.125 and $125 \mathrm{ng} / \mathrm{ml}$ (reprinted with permission from Ref. [7], IEEE/OSA 
important a fast detection of hydrogen at low concentrations. It is well known that, when a palladium (Pd) or $\mathrm{Pd}$-alloy film is exposed to hydrogen $(\mathrm{H})$, it is converted to $\mathrm{PdH}$ with changing refractive and absorption indexes of a resulting composite material [13-15]. Such changes are possible to monitor at using the developed PCF tapers $[6,16]$. For fast detection of low hydrogen concentrations, an eight nanometer palladium film was deposited on the waist of a tapered PCF (with $\rho_{W}=$ $28 \mu \mathrm{m}$ ) over a length $L_{0}=10 \mathrm{~mm}$. The film was deposited in a high-vacuum chamber by thermal evaporation. To make the SM-PC-SM fiber transmission spectrum measurements, we used an LED source with a central wavelength of $1280 \mathrm{~nm}$ (with $80 \mathrm{~nm}$ of spectral width) and an optical spectrum analyzer (OSA), Ando AQ-6315E. Measured transmission spectra of the taper with an $8 \mathrm{~nm}$ thick palladium film, which was placed in a nitrogen atmosphere with a hydrogen concentration between 1.2 and 5.6 vol\%, are presented in Fig. 4.

It is seen in Fig. 4 that, when the device was exposed to hydrogen concentrations 1.2 to $5.6 \mathrm{vol} \%$, the intensity of the transmitted spectrum peaks increases in a nonlinear manner. The sensor response time was approximately $10 \mathrm{~s}$. Also, a noticeable shift in the output spectrum patterns is absent. It is necessary to point out that at hydrogen concentration more than $6 \%$ the transmitted spectrum is similar to that at $5.6 \%$. It means that saturation of the $8 \mathrm{~nm}$ thick palladium film takes place. To explain the behavior of the transmission spectra, it was proposed an electrodynamics model accounting for diffraction transformation of local waist modes [17]. Calculation results for the spectral taper transmittance obtained by taking into account $m \geq 9$ local waist modes are presented in Fig. 5.

It is seen in Fig. 5 that an increase of the hydrogen concentration leads to a growth of integral transmittance of the sensor and practically does not affect wavelength positions of transmittance extrema. In such conditions, it is interesting to optimize the sensor from the standpoint of maximizing the sensitivity of its integral transmittance $P$ to a hydrogen concentration $C$. We investigated possibilities of a sensor optimization by changing the length of the taper waist $L_{O}$. Figure 6 shows the calculated normalized sensor transmittance versus $C$ for experimental and optimal $L_{O}$ (left) and a sensor sensitivity $(d P / d C)_{C=O}$ versus waist length $L_{O}$ (right). It is possible to see in Fig. 6 (left) that the intensity at the output of the sensor is monotonously increasing function of the hydrogen concentration and calculated results are in good accordance with experimental ones (circles). It is possible also to see in Fig. 6 (right) that at $L_{O}=6.375 \mathrm{~mm}$ exists a sensitivity maximum for the investigated sensor. Besides, in the transition from the experimental waist length $L_{0}=10.00 \mathrm{~mm}$ to the optimal value $L_{0}=6.375 \mathrm{~mm}$, both the transparency and sensitivity of the sensor essentially grow. The existence of a clearly expressed maximum sensitivity of the sensor at $L_{0}=6.375 \mathrm{~mm}$ follows from Fig. 6 (right).

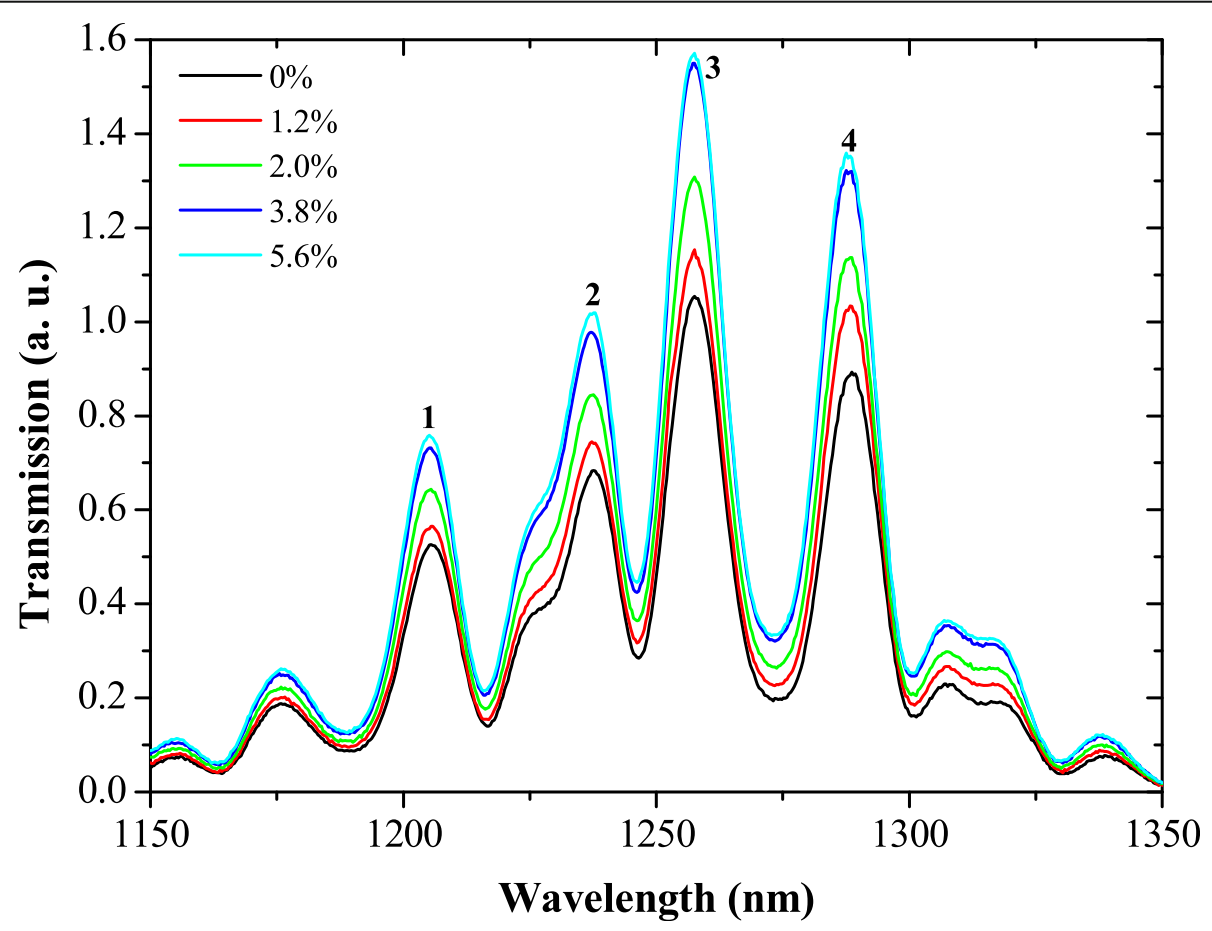

Fig. 4 Transmission spectra of the investigated taper with an $8 \mathrm{~nm}$ thick palladium film at different hydrogen concentrations in a nitrogen atmosphere (reprinted with permission from Ref. [16], OSA 

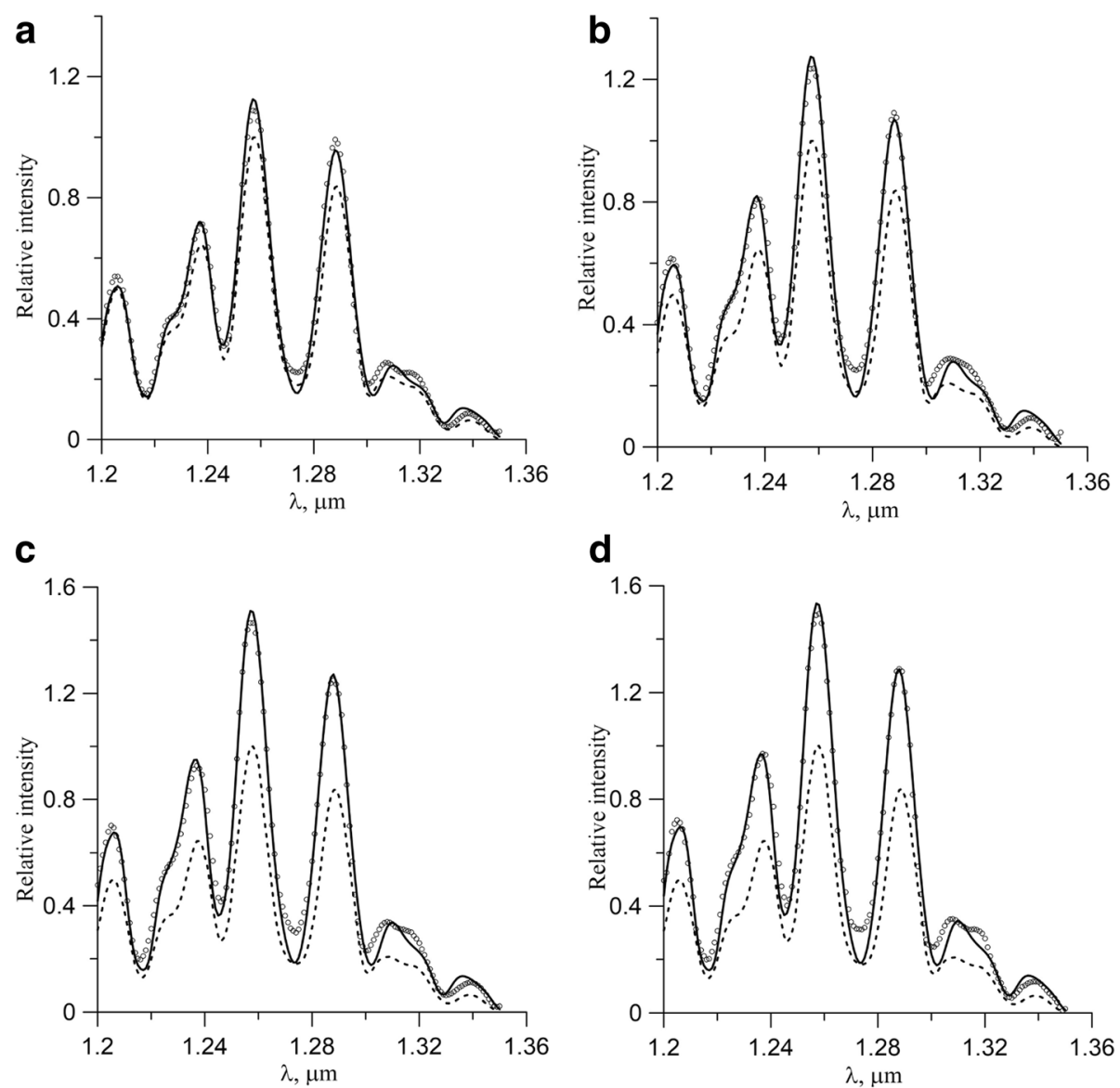

Fig. 5 Calculated results of a relative intensity at the taper output at different hydrogen concentrations (volume \%): (a) 1.2\%, (b) 2.0\%, (c) 3.8\%, and (d) 5.6\% (solid curves); circles are obtained with experiments, dashed lines indicate, for comparison, an interpolation of experimental data at a zero hydrogen concentration
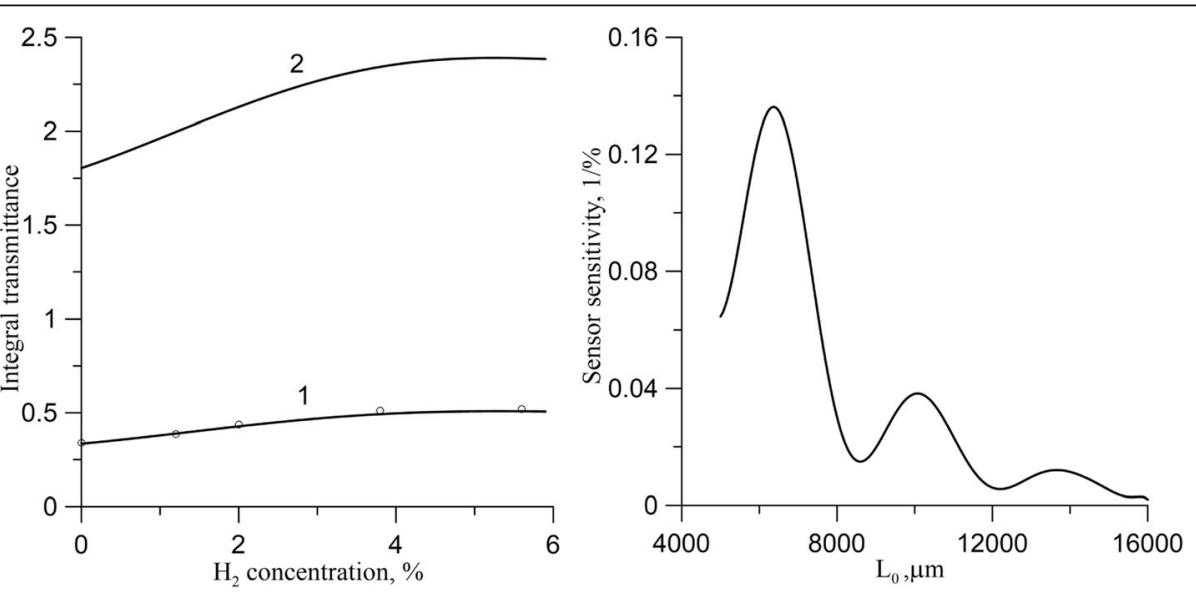

Fig. 6 Calculated integral transmittance of the investigated hydrogen sensor versus a hydrogen concentration (left) and a sensor sensitivity for different waist lengths $L_{0}$ (right). Curves 1 and 2 are calculation results for lengths $L_{0}=10.00 \mathrm{~mm}$ and $6.374 \mathrm{~mm}$, respectively. Circles are experimental results 


\section{Conclusions}

In this paper, we report on compact photonic crystal fiber Mach-Zehnder modal interferometers based on no adiabatically tapered special silica fibers, which were coated with an ultra-thin Bovine Serum Albumin (BSA) antigen or an $8 \mathrm{~nm}$ palladium film to detect, respectively, the interaction between the BSA antigen and an anti-BSA antibody with a record detection limit of 125 $\mathrm{pg} / \mathrm{ml}$ of the antibody concentration or for fast detection of low hydrogen concentrations, between 1.2 and 5.6 vol\%. Using a previously developed electrodynamics model, accounting for diffraction transformation of local modes in the taper coated with the $8 \mathrm{~nm}$ palladium film, we interpreted obtained experimental data. It was confirmed that an increase of a hydrogen concentration in a nitrogen atmosphere between 1.2 and $5.6 \mathrm{vol} \%$ leads to an increase of integral transmittance of the investigated hydrogen sensor and practically does not affect wavelength positions of transmittance maxima. An optimal taper sensing length $L_{O}=6.375 \mathrm{~mm}$ was determined for the investigated hydrogen sensor.

\begin{abstract}
Acknowledgements
Sotsky A.B. would like to thank a Byelorussian State Scientific Program "Photonics 1.3.03" for partially support of this work. The authors also would like to thank their colleagues Villatoro, J., Monzón-Hernandez, D., Kir'yanov, A.V., Calixto, S., Sotskaya, L.I., Badenes, G., Betancur-Ochoa, J.E., and MontagutFerizzola, Y.J. for productive scientific cooperation.
\end{abstract}

\section{Funding}

Declared at acknowledgements.

\section{Availability of data and materials}

Data sharing is not applicable to this article as no datasets were generated.

\section{Authors' contributions}

Conceived and designed the experiments: VPM, ABS. Performed the experiments: VPM. Analyzed the data: VPM, ABS. Contributed analysis tool: ABS. Wrote the paper: VPM. Both authors read and approved the final manuscript.

\section{Competing interests}

The authors declare that they have no competing interests.

\section{Publisher's Note}

Springer Nature remains neutral with regard to jurisdictional claims in published maps and institutional affiliations.

\section{Author details}

${ }^{1}$ Centro de Investigaciones en Optica, Division of Photonics, Loma del Bosque 115, Colonia Lomas del Campestre, 37150, Leon, Guanajuato, Mexico. ${ }^{2}$ Dept. of Physics, A.A. Kuleshov Mogilev State University, 1 Kosmonavtov Str, 212009 Mogilev, Belarus.

Received: 28 February 2019 Accepted: 25 April 2019

Published online: 08 May 2019

\section{References}

1. Knight, J.C, Birks, T.A, Russell, P.St.J., Atkin, D.M.: All-silica single-mode optical fiber with photonic crystal cladding. Opt. Lett, 21(19),1547-1549(1996), https://doi.org/10.1364/OL.21.001547

2. Minkovich, V.P., Villatoro, J., Monzón-Hernández, D., Calixto, S., Sotsky, A.B., Sotskaya, L.I.: Holey fiber taper with resonance transmission for highresolution refractive index sensing. Opt. Express. 13(19), 7609-7614 (2005). https://doi.org/10.1364/OPEX.13.007609
3. Minkovich, V.P., Monzón-Hernández, D., Villatoro, J., Sotsky, A.B., Sotskaya, L.I.: Modeling of holey fiber tapers with selective transmission for sensor applications. J. Lightw. Technol. 24(11), 4319-4328 (2006). https://doi.org/10. 1109/JLT.2006.884207

4. Villatoro, J., Minkovich, V.P., Monzón-Hernández, D.: Temperatureindependent strain sensor made of tapered holey optical fibers. Opt. Lett. 31(3), 305-307 (2006). https://doi.org/10.1364/OL.31.000305

5. Minkovich, V.P., Kir'yanov, A.V., Sotsky, A.B., Sotskaya, L.I.: Large-mode-area holey fibers with a few air channels in cladding: modeling and experimental investigation of modal properties. J. Opt. Soc. Am. B. 21(6), 1161-1169 (2004). https://doi.org/10.1364/JOSAB.21.001161

6. Minkovich, V.P., Villatoro, J., Sotsky, A.B.: Tapered photonic crystal fibers coated with ultra-thin films for highly sensitive bio-chemical sensing. In: Abstracts of the European Optical Society Biennial Meeting 2018, TU Deft Aula Conference Center, Deft, Netherlands, 8-12 October 2018, 9, 446-447 (2018)

7. Betancur-Ochoa, J.E., Minkovich, V.P., Montagut-Ferizzola, Y.J.: Special photonic crystal modal interferometer for highly sensitive biosensing. J. Lightw. Technol. 35(21), 4747-4751 (2017). https://doi.org/10.1109/JLT.2017.2761738

8. Li, X., Liu, L., Zhao, J., Tan, J.: Optical properties of sodium chloride solution within the spectral range from 300 to $2500 \mathrm{~nm}$ at room temperature. Appl. Spectroscopy. 69(5), 635-640 (2015). https://doi.org/10.1366/14-07769R

9. Nagel, T., Ehrentreich-Forster, E., Singh, M., Schmitt, K., Brandenburg, A., Berka, A., Bier, F.F.: Direct detection of tuberculosis infection in blood serum using three optical label-free approaches. Sensors Actuators B Chem. 129(2), 934-940 (2008). https://doi.org/10.1016/j.snb.2007.10.009

10. Yadav, T.K., Narayasnaswamy, R., Abu Bacar, M.H., Mustapha Kamil, Y., Mahdi, M.A.: Single-mode tapered fiber-optic interferometer based refractive index sensor and its application to protein sensing. Opt. Express. 22(19), 2280222807 (2014). https://doi.org/10.1364/OE.22.022802

11. Juan Hu, D.J., Lim, J.L., Park, M.K., Kao, L.T.-H., Wang, Y., Wei, H., Tong, W.: Photonic crystal fiber-based interferometric biosensor for streptavidin and biotin detection. IEEE J. Sel. Topics Quantum Electron. 18(4), 1293-1297 (2012). https://doi.org/10.1109/JSTQE.2011.2169492

12. Yu, W., Lang, T., Bian, J., Kong, W.: Label-free fiber optic biosensor based on thin-core modal interferometer. Sens. Actuators B: Chem. 228, 322-329 (2016). https://doi.org/10.1016/j.snb.2016.01.029

13. Silva, S.F., Coelho, L., Frazão, O., Santos, J.L., Malcata, F.X.: A review of palladium-based fiber-optic sensors for molecular hydrogen detection. IEEE Sensors J. 12(1), 93-102 (2012). https://doi.org/10.1109/JSEN.2011.2138130

14. Butler, M.A.: Optical fiber hydrogen sensor. Appl. Phys. Lett. 45(10), 10071009 (1984). https://doi.org/10.1063/1.95060

15. Zhao, Z., Carpenter, M.A., Xia, H., Welsh, D.: All-optical hydrogen sensor based on a high alloy content palladium thin film. Sensors Actuators B Chem. 113(1), 532-538 (2006). https://doi.org/10.1016/j.snb.2005.03.070

16. Minkovich, V.P., Monzón-Hernández, D., Villatoro, J., Badenes, G.: Microstructured optical fiber coated with thin films for gas and chemical sensing. Opt. Express. 14(18), 8413-8418 (2006). https://doi.org/10.1364/OE.14.008413

17. Minkovich, V.P., Sotsky, A.B., Shilov, A.V., Sotskaya, L.I.: Taper with palladium coating in photonic crystal fiber as a sensitive element of hydrogen sensor. J. Appl. Spectrosc. Accepted for publication in 2019

\section{Submit your manuscript to a SpringerOpen ${ }^{\circ}$ journal and benefit from:}

- Convenient online submission

- Rigorous peer review

- Open access: articles freely available online

- High visibility within the field

- Retaining the copyright to your article

Submit your next manuscript at $>$ springeropen.com 